\title{
Exposição de Adolescentes à Violência em Diferentes Contextos: Relações com a Saúde Mental
}

\author{
Naiana Dapieve Patias ${ }^{1}$ \\ Universidade Federal do Rio Grande do Sul, Porto Alegre, RS, Brasil, \\ Faculdade Meridional - IMED, Passo Fundo, RS, Brasil \\ Doralúcia Gil da Silva \\ Débora Dalbosco Dell'Aglio \\ Instituto de Psicologia da Universidade Federal do Rio Grande do Sul, \\ Porto Alegre, RS, Brasil
}

\section{Resumo}

Este estudo teve como objetivo discutir a questão da exposição de adolescentes à violência em diferentes contextos e suas relações com a saúde mental. Por meio de uma revisão sistemática nas bases SciELO (Scientific Eletronic Library Online), PsycINFO e Medline, de 2009 a 2013, foram recuperados 19 artigos, a partir de critérios de exclusão e inclusão. Foram utilizadas categorias definidas a priori a partir de três eixos principais de análise: (a) Características metodológicas; (b) Tipo de violência e contexto de investigação; e (c) Repercussões da violência na saúde mental dos adolescentes. A violência tanto na família como em outros contextos do desenvolvimento produz consequências negativas na saúde mental dos adolescentes. Observa-se a necessidade de investimento em prevenção e promoção de saúde mental na adolescência, além de intervenções na busca da prevenção à exposição à violência através da identificação dos fatores de risco e de proteção nos vários contextos de desenvolvimento dos adolescentes.

Palavras-chave: Adolescência, violência, saúde mental.

\section{Exposure of Adolescents to Violence in Different Contexts: Relationships with Mental Health}

\begin{abstract}
This study aimed to discuss the issue of exposure of adolescents to violence in different contexts and their relationships with mental health. Through a systematic review of the SciELO (Scientific Eletronic Library Online), MEDLINE and PsycINFO, from 2009 to 2013, 19 articles were recovered from inclusion and exclusion criteria. Categories defined a priori were used from three main areas of analysis: (a) methodological characteristics; (b) type of violence and context of investigation and (c) impact of violence on the mental health of adolescents. The violence in the family and in other developmental contexts produces negative effects on the mental health of adolescents. This study highlights the need for investment in prevention and promotion of mental health in adolescence, as well as interventions in pursuit of preventing exposure to violence through identification of risk and protective factors in the various contexts of adolescent development.
\end{abstract}

Keywords: Adolescence, violence, mental health.

Endereço para correspondência: Rua Ramiro Barcelos, 2600, sala 105, Bairro Santa Cecília, Porto Alegre, RS, Brasil 90035-003. E-mail: naipatias@hotmail.com,doralu.gil@gmail.com e debora.dellaglio@ufrgs.br 


\section{Exposición de los Adolescentes a la Violencia en Diferentes Contextos: Las Relaciones con la Salud Mental}

\section{Resumen}

Este estudio tuvo como objetivo discutir la cuestión de la exposición de los adolescentes a la violencia en diferentes contextos y su relación con la salud mental. Través de una revisión sistemática de la SciELO (Scientific Eletronic Library Online), MEDLINE y PsycINFO, de 2009 y 2013, 19 artículos fueron recuperados de los criterios de inclusión y exclusión. Fueron utilizadas categorías definidas a priori a partir de tres ejes principales de análisis: (a) las características metodológicas; (b) tipo de violencia y el contexto de la investigación; y (c) Impacto de la violencia en la salud mental de los adolescentes. La violencia en la familia y en otros contextos de desarrollo produce efectos negativos sobre la salud mental de los adolescentes. Observe la necesidad de invertir en la prevención y promoción de la salud mental en la adolescencia, así como las intervenciones en la búsqueda de la prevención de la exposición a la violencia a través de la identificación de factores de riesgo y de protección en los diferentes contextos de desarrollo de los adolescentes.

Palabras clave: Adolescencia, violencia, salud mental.

A adolescência é um importante período do desenvolvimento no qual ocorrem muitas transformações biopsicossociais, e surgem novas habilidades, busca de oportunidades, exploração e descobertas (Steinberg \& Lerner, 2004). Os processos de independização e autonomia, característicos dessa etapa, propiciam um esperado afastamento do jovem em relação às figuras parentais, na tentativa de construir e exercer seu novo posicionamento frente ao mundo (Barbosa \& Wagner, 2013; Steinberg, 2000, 2005). Ao voltar-se para o meio social, buscar o grupo de pares e estar aberto a novas oportunidades e experiências, o adolescente tende a estar mais exposto a diferentes situações que podem colocá-lo em risco e vulnerabilidade (Benetti et al., 2006). De fato, em uma abordagem sistêmica, considera-se que a adolescência e seus riscos dependem de vários aspectos, não só da pessoa (individuais), mas também dos contextos em que $o$ adolescente está inserido, tanto micro (família, escola, comunidade), como macrossistema (cultura, valores, etc; Ministério da Saúde, 2010a).

Diante disso, destaca-se que o contexto no qual o adolescente está inserido como, por exemplo, a família, escola ou comunidade - desempenha papel importante para o seu desenvolvimento, exercendo função de proteção (Braga \& Dell'Aglio, 2012; Guzmán, 2007). Entretanto, nem sempre os contextos de desenvolvimento cumprem esse papel, como por exemplo, quando há exposição à violência. Pesquisas nacionais e internacionais vêm apontando altos índices de exposição a diversos tipos de violência entre os adolescentes que vivem em diferentes contextos (Braga \& Dell'Aglio, 2012; Haynie, Petts, Maimon, \& Piquero, 2009; Malta et al., 2010; Ministério da Saúde, 2011; Zavaschi, Benetti, Polanczyk, Soles, \& Sanchotene, 2002) sendo que a vitimização nessa faixa etária é maior se comparada com crianças e adultos. Dados do Mapa de violência do Brasil (Waiselfisz, 2012) revelam que as mortes de crianças e adolescentes por causas externas (acidentes e violência, geralmente homicídios) têm aumentado se comparadas com as mortes por causas naturais (doenças, por exemplo) que diminuíram de 1980 até 2010.

A World Health Organization (WHO, 2002) define violência como sendo a utilização da força física, do poder ou ameaça que resulta em dano, morte, privação ou dano psicológico, contra si mesmo ou contra outra pessoa. Segundo essa definição, a violência pode ser dividida segundo seus tipos específicos, a saber: violência autodirecionada (voltada para si mesmo suicídio, por exemplo), interpessoal (violência intra e extrafamiliar) e coletiva (violência social, política e econômica, geralmente cometida por 
grandes grupos de indivíduos ou por estados - crimes, terrorismo, por exemplo). Quanto às características do ato violento, essa definição considera a violência física, psicológica, sexual e negligência.

Neste trabalho, será dada ênfase à violência interpessoal definida a partir do seu contexto de ocorrência: dentro da família (intrafamiliar), ou fora dela (extrafamiliar). A violência intrafamiliar é entendida como um padrão de relacionamento violento envolvendo ações ou omissões que são perpetradas por alguém que mantenha laços significativos (consanguíneos ou afetivos) com a vítima (Habigzang \& Caminha, 2004). Já a violência extrafamiliar ocorre quando o agressor não pertence ao âmbito das relações familiares (Habigzang \& Caminha, 2004), e, em geral, ocorre em locais frequentados pelos jovens (escola, bairro, trânsito, locais de trabalho, instituições de saúde, entre outros). A violência fora do âmbito familiar é conhecida também como violência comunitária, a qual, da mesma forma, envolve violência no contexto social ou ambiental do indivíduo, podendo haver agressões, assaltos, estupros, roubos, com ou sem presença de armas (WHO, 2002).

No que diz respeito aos efeitos da violência, de modo geral, são negativos e inúmeros. Podem cessar, impedir ou retardar o desenvolvimento social, cognitivo e emocional, ou ainda potencializar o risco para psicopatologias (Koller \& De Antoni, 2004). De fato, a violência pode trazer consequências físicas, psíquicas e sociais. Dentre as principais consequências da violência, estão aquelas relativas à saúde mental, entendida como um completo bem-estar físico, mental e social e não meramente a ausência de doenças. Está relacionada com a promoção do bem-estar, a prevenção dos transtornos mentais, tratamento e reabilitação de pessoas afetadas por transtornos mentais (WHO, 2010).

Em relação às doenças mentais, a violência tem aumentado o risco de desenvolvimento de transtornos mentais que podem ser desencadeados a curto, médio e longo prazo. Entre os principais transtornos mentais que resultam da violência, estão: Transtorno de Estresse Pós-Traumático (TEPT), transtornos de ansiedade, transtornos de humor (especialmente a depressão) e transtornos de personalidade (Mrug \& Windle, 2010). Relatório da WHO (2014) revela que houve, em 2012, mais de um milhão de mortes de adolescentes decorrentes, principalmente, de problemas de saúde mental e da violência.

Tanto a violência como os problemas de saúde mental representam riscos ao desenvolvimento dos adolescentes, embora nem sempre sejam priorizados nas intervenções ou nas políticas públicas (Ministério da Saúde, 2010a, 2010b). Dessa forma, neste trabalho, procura-se aprofundar o conhecimento e as relações entre os dois problemas de saúde pública relacionados ao desenvolvimento dos adolescentes, respondendo às seguintes questões: Quais os contextos de exposição à violência na adolescência que têm sido investigados? Quais os tipos de violência que ocorrem nesses contextos? Quais os principais métodos empregados no estudo da exposição à violência na adolescência? Quais as relações da violência, em diferentes contextos, com a saúde mental de adolescentes?

\section{Método}

Este estudo de revisão sistemática da literatura teve por objetivo caracterizar estudos sobre a exposição à violência em diversos contextos e suas relações com a saúde mental em adolescentes. Segundo Sampaio e Mancini (2007), a revisão sistemática é um tipo de pesquisa que utiliza a literatura sobre determinado tema como uma fonte de dados. A revisão sistemática é uma ferramenta que permite organizar, analisar criticamente e sintetizar resultados de estudos formulando-se perguntas e fazendo a identificação, seleção e avaliação crítica de estudos científicos contidos nas bases de dados (Lopes \& Fracolli, 2008; Zoltowski, Costa, Teixeira, \& Koller, 2014). De acordo com Sampaio e Mancini (2007), é importante a elaboração de um protocolo de pesquisa que inclua os artigos encontrados, critérios de inclusão e exclusão, definição dos desfechos de interesse, verificação dos resultados e análise dos resultados.

As etapas seguidas nessa revisão sistemática foram cinco, de acordo com a proposta metodo- 
lógica de revisão sistemática de Sampaio e Mancini (2007): a primeira envolveu a definição da pergunta de pesquisa. A segunda etapa dizia respeito à busca dos estudos, certificando-se de que os artigos importantes do tema fossem incluídos na revisão. Para tanto, a definição das palavras-chave, estratégias de busca e definição das bases de dados para a busca foram critérios importantes. A busca dos documentos (Steinberg \& Lerner, 2004) foi realizada a partir de três bases de dados, tanto nacionais como internacionais, a saber: (a) SciELO (Scientific Eletronic Library Online), (b) PsycINFO e (c) Medline (Pubmed). Foi utilizada a seguinte combinação de descritores, no campo assunto, para a busca dos artigos: violence AND (adolescen*) AND (school OR community OR family) AND mental health. Os descritores foram incluídos a partir do objetivo geral do estudo que foi verificar a exposição à violência em vários contextos do desenvolvimento e suas repercussões na saúde mental dos adolescentes. Desta forma, a saúde mental foi incluída como palavra chave. A fim de verificar as repercussões da violência na saúde mental, os artigos selecionados foram lidos na íntegra enfocando-se este aspecto. Primeiramente, foi realizada a busca de trabalhos em cada base de dados sem delimitação de ano. A combinação de descritores foi colocada no campo geral de cada base, com a busca de descritores em "todos os campos", incluindo-se a procura por essa combinação de descritores no título, resumo ou ao longo do documento. Foram recuperados 4.691 documentos, sendo 32 na base SciELO, 1.953 Medline (Pubmed) e 2.706 na base PsycINFO.

$\mathrm{Na}$ etapa seguinte que envolvia a revisão e seleção dos estudos, foi realizada uma identificação inicial dos artigos através dos títulos e resumos (abstracts), de forma independente por dois pesquisadores, seguindo os critérios de inclusão: (a) publicação dos últimos cinco anos (2009-2013), (b) artigo empírico escrito em inglês, português ou espanhol; e critérios de exclusão: (a) documentos que não eram artigos (teses, dissertações, editoriais e livros), (b) artigos que não estavam disponíveis em texto completo e (c) artigos repetidos entre as bases de dados. Nesta etapa, 4.588 artigos foram excluídos, restando
113 para leitura na íntegra. Quando o título e resumo não foram suficientes, fez-se necessário a busca e leitura do trabalho na íntegra. Com a leitura dos artigos selecionados nessa primeira etapa, utilizaram-se outros critérios de exclusão a fim de atingir aos objetivos propostos: (a) aqueles artigos que possuíam, nas amostras, crianças e adolescentes, não diferenciando os resultados por idades e, (b) artigos que não tinham por objetivo ou foco principal, relacionar violência com saúde mental. Nessa etapa, 94 artigos foram excluídos (Figura 1), restando apenas 19 artigos na análise final, sendo três da base SciELO, 11 Medline (Pubmed) e cinco da base PsycINFO.

$\mathrm{Na}$ quarta etapa analisou-se a metodologia dos estudos e as relações entre violência e saúde mental na adolescência apresentadas nos resultados das diferentes pesquisas. $\mathrm{Na}$ quinta e última etapa, foi realizada a análise de conteúdo dos artigos, que levou à organização dos resultados em três categorias: (a) Características metodológicas; (b) Tipo(s) de violência (direta e indireta) e contextos de investigação; e (c) Repercussões da violência na saúde mental dos adolescentes.

\section{Resultados}

\section{Características Metodológicas}

Nesta categoria são apresentadas as informações quanto ao delineamento dos estudos, características dos participantes, instrumentos e análise dos dados. As amostras variaram de 152 a 3.614 adolescentes, com idades entre 10 a 19 anos. A maioria dos estudos foi realizada com adolescentes de escolas públicas de vários países, incluindo Brasil, Peru, Vietnã e Estados Unidos, que viviam com suas famílias, como por exemplo, no estudo de Benetti, Pizetta, Schwartz, Hass, e Melo (2010) - e adolescentes que viviam em instituições de acolhimento ou na rua, como por exemplo, no estudo de Haber e Toro (2009). Predominaram estudos internacionais, tendo sido selecionado apenas um estudo nacional (Benetti et al., 2010), demonstrando, dessa forma, a necessidade de maior investigação da temática da violência relacionada à saúde mental no contexto brasileiro. 


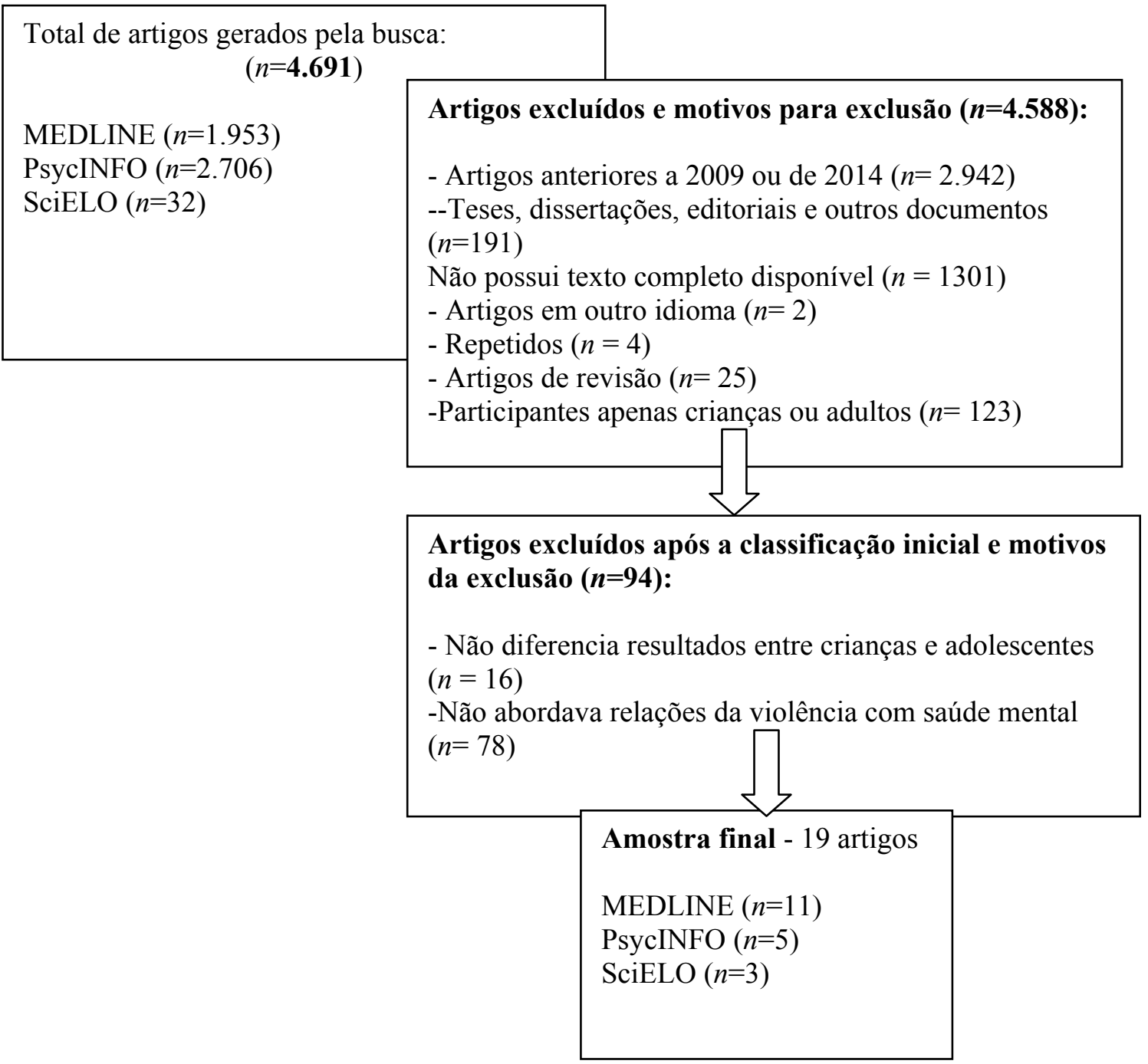

Figura 1. Etapas de exclusão de artigos.

Quanto ao delineamento, grande parte dos estudos é transversal, embora quatro estudos sejam longitudinais (Betancourt, Agnew-Blais, Gilman, Williams, \& Ellis, 2010; Betancourt, Brennan, Rubin-Smith, Fitzmaurice, \& Gilman, 2010; Haber \& Toro, 2009; Hasanović et al., 2009). Quanto aos instrumentos de coleta de dados, predominou o uso de escalas e testes ou questionários estruturados, sendo poucos os estudos que utilizaram perguntas abertas ou entrevistas com os participantes (Cano, Gutiérrez, \& Nizama, 2009; Wigderson \& Lynch, 2013). Dentre os principais instrumentos utilizados para investigar exposição à violência em diversos contextos, estão: Conflict Tatics Scales (CTS), Children's Report of Exposure to Violence e Exposure to Community Violence. Os instrumentos mais utilizados para investigar saúde mental foram: versões diferentes do Post-Traumatic Stress Disorder (PTSD) Symptom Scale, Child Behavior Checklist (CBCL), Children's Depression Inventory (CDI) e Youth Self Report (YSR).

A análise dos dados, da maioria dos artigos, foi quantitativa, com a utilização de análises estatísticas descritivas, análises fatoriais, regressão linear e múltipla e modelos multivariados e estruturais. Entretanto, um estudo utilizou a metodologia de análise de dados qualitativa, através da análise de conteúdo por temas (Eggerman \& Panter-Brick, 2010).

\section{Tipo(s) de Violência e Contexto de Investigação}

Os estudos variaram quanto ao tipo de violência investigada, considerando os diversos contextos de ocorrência da violência - família, 
escola ou comunidade. Alguns estudos tiveram como foco a violência intrafamiliar (Haber \& Toro, 2009), extrafamiliar (Benetti et al., 2010; Epstein-Ngo, Maurizi, Bregman, \& Ceballo, 2013; Lambert, Cammack, Boyd, \& Ialongo, 2012), e ambos (Jenkins, Turner, \& Wang, 2009; Nguyen, Dunne, \& Vu Le, 2010), passando pela exposição à cyber violência ou violência tecnológica (Wigderson \& Lynch, 2013) até a violência autodirigida (contra si mesmo) e contra os outros (Cano et al., 2009). Cabe ressaltar que, embora todos os estudos apontem para uma direção mais específica pela qual investiga a exposição à violência, a maioria deles destaca o complexo interjogo entre a(s) violência(s) dos vários contextos e como a exposição em um contexto torna o adolescente vulnerável à exposição em outros contextos.

No que diz respeito às formas de violência, as investigações centraram-se na exposição direta, quando o adolescente é a própria vítima da violência (Nguyen et al., 2010; Wigderson \& Lynch, 2013), mas também na exposição à violência de forma indireta, quando o adolescente testemunha a violência nos vários contextos (Bach \& Louw, 2010; Copeland-Linder, Lambert, \& Ialongo, 2010; Epstein-Ngo et al., 2013; Jenkins et al., 2009; Voisin \& Neilands, 2010; Zinzow et al., 2009). Ainda houve pesquisas que tiveram por objetivo investigar exposição a eventos traumáticos, eventos estressores e experiências de guerra (Al-Krenawi, Graham, \& Kanat-Maymon, 2009; Betancourt, Agnew-Blais, et al., 2010; Betancourt, Brennan, et al., 2010; Eggerman \& Panter-Brick, 2010; Hasanović et al., 2009; Rheingold, Zinzow, Hawkins, Saunders, \& Kilpatrick, 2012). Além disso, outro estudo investigou ambas as formas de exposição à violência (direta e indireta; Benetti et al., 2010). Um deles analisou a violência dos adolescentes dirigida aos outros, correlacionada com a violência autodirecionada (tentativa de suicídio; Cano et al., 2009).

Quanto ao tipo de violência, no geral, todas as formas de violência foram investigadas: violência autodirecionada (tentativa de suicídio) (Cano et al., 2009) e violências interpessoais, tais como violência física (Benetti et al., 2010) psicológica (Walsh et al., 2013) e sexual (Houck, Nugent, Lescano, Peters, \& Brown, 2010). Alguns estudos contemplaram somente um tipo de violência, enquanto outros investigaram mais de um tipo (Nguyen et al., 2010).

\section{Repercussões da Violência na Saúde Mental dos Adolescentes}

No geral, todos os artigos identificaram alta prevalência de exposição à violência nos vários contextos do desenvolvimento do adolescente. Além disso, as investigações ressaltaram as consequências negativas dos vários tipos de exposição e formas de violência, na saúde mental dos adolescentes, independente do contexto de desenvolvimento, embora alguns estudos tenham encontrado maior associação quando a exposição é intrafamiliar (Benetti et al., 2010; Nguyen et al., 2010). A maioria dos estudos analisou a saúde mental através da investigação de sintomas de doença mental ou de instrumentos padronizados que incluíam sintomas descritos no DSM-IV, como por exemplo, depressão (Benetti et al., 2010; Jenkins et al., 2009; Nguyen et al., 2010), ansiedade (Benetti et al., 2010; Jenkins et al., 2009; Nguyen et al., 2010), somatização (Jenkins et al., 2009), TEPT (Hasanović et al., 2009; Jenkins et al., 2009; Rheingold et al., 2012; Voisin \& Neilands, 2010; Zinzow et al., 2009), uso de álcool e outras drogas (Haber \& Toro, 2009; Rheingold et al., 2012) e comportamento sexual de risco (Houck et al., 2010; Voi$\sin \&$ Neilands, 2010). Além disso, grande parte dos estudos utilizou a categorização sintomas de internalização (depressão e ansiedade) e externalização (agressão) como indicadores de saúde mental (Al-Krenawi et al., 2009; Benetti et al., 2010; Betancourt, Agnew-Blais, et al., 2010; Copeland-Linder et al., 2010; Haber \& Toro, 2009; Jenkins et al., 2009).

De modo geral, os resultados apontaram que a violência esteve associada com o aumento dos sintomas de transtornos mentais, ideação suicida, vivências traumáticas, desajustamento psicossocial, problemas externalizantes, e comportamento agressivo (Benetti et al., 2010; Betancourt, Agnew-Blais, et al., 2010; Betancourt, Brennan, et al., 2010; Cano et al., 2009; Cope- 
land-Linder et al., 2010; Haber \& Toro, 2009; Lambert et al., 2012; Rheingold et al., 2012). Alguns estudos relataram diferenças observadas entre os sexos relacionadas à violência e suas consequências para a saúde mental, tais como: maior uso de armas e violência física entre meninos (Walsh et al., 2013); testemunhar violência esteve mais associado a comportamentos agressivos em meninos (Lambert et al., 2012); maior exposição à violência comunitária em meninos (Voisin \& Neilands, 2010), assim como maiores níveis de depressão em meninas (Bach \& Louw, 2010). Outros estudos apresentaram a exposição à violência como preditora de consequências, tais como, TEPT, depressão, comportamentos internalizantes e externalizantes, problemas de autoestima e ansiedade (Jenkins et al., 2009; Nguyen et al., 2010). Um estudo com jovens americanos que frequentavam escolas alternativas e terapêuticas observou maior probabilidade de adolescentes expostos ao abuso sexual apresentarem sintomatologia significativa para diagnósticos psiquiátricos, bem como para comportamento sexual de risco (Houck et al., 2010). Em um estudo experimental, realizado com adolescentes sobreviventes da guerra da Bósnia e Herzegovina, foi observado que as meninas apresentaram mais sintomas de TEPT do que os meninos, mas que houve redução de sintomas entre os participantes de um projeto de intervenção (Hasanović et al., 2009). Ainda, um levantamento com uso de questionários realizado com 388 adolescentes nos Estados Unidos, abordou a cyber vitimização, a qual esteve negativamente relacionada com aspectos do bem-estar do adolescente e desempenho acadêmico (Wigderson \& Lynch, 2013).

Outros estudos, em menor quantidade, abordaram aspectos positivos, como por exemplo, as estratégias de coping, esperança e resiliência ante a exposição à violência (Eggerman \& Panter-Brick, 2010; Epstein-Ngo et al., 2013) ou a autoestima de adolescentes expostos à violência (Nguyen et al., 2010; Wigderson \& Lynch, 2013). Foram observadas correlações negativas entre esses constructos e a violência. Já no estudo sobre coping (Epstein-Ngo et al., 2013), aspectos protetivos que podem mitigar os riscos da exposição à violência foram destacados. Além disso, os autores desse grupo de trabalhos sugerem uma abordagem de maior alcance em relação ao conceito de saúde mental, considerando-o não apenas como ausência de doença - como nos estudos que abordam apenas sintomas de doença mental - mas também incluindo aspectos que podem interferir no bem-estar do adolescente.

\section{Discussão}

Muitos dos estudos realizados em contexto internacional ressaltam aspectos importantes da violência tanto direta quanto indireta, em vários contextos do desenvolvimento - família, escola, comunidade, acolhimentos institucionais, e sua relação com a saúde mental dos adolescentes. Entre os aspectos destacados, pode-se observar que os estudos relatam grande exposição à violência na adolescência, sendo esses adolescentes, na maioria das vezes, vítimas. Essa exposição traz consequências negativas ao longo do desenvolvimento, como o aumento dos sintomas de transtornos mentais, ideação suicida, vivências traumáticas, desajustamento psicossocial, problemas externalizantes e internalizantes, comportamento agressivo, violência física, problemas de autoestima, ansiedade, comportamento sexual de risco, entre outros (Al-Krenawi et al., 2009; Benetti et al., 2010; Betancourt, Agnew-Blais, et al., 2010; Betancourt, Brennan, et al., 2010; Cano et al., 2009; Copeland-Linder et al., 2010; Haber \& Toro, 2009; Lambert et al., 2012). Além dos estudos que discutem o impacto negativo da violência, outras pesquisas enfocam aspectos positivos e de enfrentamento frente à violência, investigando variáveis como o bem-estar, esperança e estratégias de coping (Eggerman \& Panter-Brick, 2010; Epstein-Ngo et al., 2013; Nguyen et al., 2010; Wigderson \& Lynch, 2013), numa perspectiva teórica da Psicologia Positiva (Rutter, 1999; Yunes, 2003). Estes aspectos são essenciais servindo como fatores de proteção tanto para a violência quanto para a doença mental.

Considerando a alta exposição de adolescentes à violência no Brasil, tem sido destacada a necessidade de estudos que investiguem as 
relações entre exposição à violência e a saúde mental no contexto brasileiro (Waiselfisz, 2012), bem como suas repercussões. Embora no Brasil existam aproximadamente 21 milhões de adolescentes, os quais representam uma parcela significativa da pirâmide etária do país (United Nation Educational, Scientific and Cultural Organization [UNICEF], 2011), as políticas públicas atuais parecem não serem capazes de garantir a proteção e prevenir situações de risco ou vulnerabilidade entre os jovens (WHO, 2002). Novas investigações, direcionadas ao problema da violência nessa população, poderiam contribuir para fornecer dados empíricos, de forma a subsidiar futuras intervenções com essa população e o estabelecimento de políticas públicas mais eficazes.

Ainda assim, os levantamentos existentes realizados na realidade nacional mostram semelhanças com os dados internacionais. Por exemplo, dados do Mapa de violência de crianças e adolescentes do Brasil revelam que as mortes por causas externas (por acidentes e violência, geralmente, homicídios) têm aumentado se comparadas com as mortes por causas naturais (doenças, por exemplo), que têm decaído de 1980 até 2010 (Waiselfisz, 2012). Pesquisa Nacional de Saúde do Escolar (PeNSE; Instituto Brasileiro de Geografia e Estatística [IBGE], 2009) demonstrou que os adolescentes brasileiros estão expostos a vários tipos de violências, principalmente na escola e na família. Esses aspectos são relevantes, na medida em que apontam o outro lado da adolescência - como vítima e não como agressora. Embora os jovens, na maioria das vezes, sejam percebidos pelo seu comportamento agressivo ou desafiador, sabe-se que são as principais vítimas da violência (UNICEF, 2011). Em estudo realizado por Zappe e Dias (2012), com adolescentes em conflito com a lei, as autoras discutem o quanto esses foram expostos, em sua história de vida familiar, a episódios de violência, os quais estão relacionados à manifestação de condutas transgressoras. Ou seja, o adolescente, antes de ser agressor, na maioria das vezes, tem um desenvolvimento marcado por repetidas exposições à violência, no qual é a principal vítima. Dessa forma, pode ser observado um ciclo de violência que deveria ser combatido, em suas raízes sociais, econômicas e culturais.

Um estudo de revisão teórica indicou que a associação entre violência e juventude, quando não leva a morte, produz graves consequências, como a redução da expectativa de vida, a redução do potencial produtivo dos jovens e o comprometimento de sua saúde (Zappe \& Dias, 2010). Essa associação é importante e demonstra a necessidade de investimento em prevenção à violência nesse período de vida. Corroborando esse aspecto, a UNICEF (2011) destaca a importância do investimento em políticas públicas direcionadas à adolescência, devido às seguintes razões: (a) respeito ao direito de adolescentes serem tratados como cidadãos; (b) investir é um modo eficaz de garantir direitos e reduzir taxas de mortalidade; (c) pode acelerar a luta contra a pobreza, disparidades socioeconômicas e discriminação de gênero, já que muitas vezes, estas disparidades apresentam-se na adolescência, privando adolescentes de seus direitos e oportunidades de desenvolvimento pleno; (d) a fim de lidar com desafios atuais e com suas consequências (como violência, pobreza, crises, etc), os adolescentes devem estar equipados com habilidades e conhecimentos adequados e, (e) por fim, mas não menos importante, os adolescentes, mesmo sendo os futuros adultos, merecem reconhecimento, proteção, cuidados, oportunidades e apoio no presente, como adolescentes, da mesma forma que as crianças necessitam.

Todas as justificativas para maior investimento na adolescência são importantes, no entanto, o último aspecto chama a atenção, pois se comparados às crianças, talvez os adolescentes tenham maior necessidade de investimento em algumas áreas do desenvolvimento que têm sido deixadas de lado, como por exemplo, a saúde e, mais especificamente, a saúde mental. Atualmente, os adolescentes, em todo o mundo, são mais saudáveis do que os das gerações anteriores, no entanto, as suas vulnerabilidades podem ser explicadas, em grande parte, pelas características do próprio período do desenvolvimento, tais como assumir riscos e tentar transpor limites, podendo envolver-se em comportamentos de risco (UNICEF, 2011). Além disso, muitas 
violências que acometem crianças têm seu pico na adolescência. $\mathrm{O}$ mesmo ocorre em relação a questões relativas à saúde mental, pois muitas psicopatologias apresentam um surgimento mais marcado nesse período (WHO, 2012). De fato, muitos países têm registrado um aumento no número de casos de depressão, ansiedade e transtornos alimentares, induzidos, frequentemente, pela exposição à violência na adolescência (Avanci, Assis, Oliveira, Ferreira, \& Pesce, 2007; Bahls \& Bahls, 2002; Ministério da Saúde, 2010a, 2010b; Rocha, Zeni, Caetano, \& Kieling, 2013; UNICEF, 2011).

Em termos de políticas públicas para a saúde do adolescente, no Brasil, tem-se o Programa Saúde do Adolescente (PROSAD) que foi o primeiro programa criado para atender a população de 10 a 19 anos, em 1989. Tem, como meta, intervir na prevenção de doenças e promoção da saúde integral do adolescente, contemplando áreas temáticas como o crescimento e desenvolvimento, sexualidade, saúde bucal, mental, reprodutiva, escolar, prevenção de acidentes, violência, família, trabalho, cultura, esporte e lazer (Ministério da Saúde, 1996). Apesar de, no papel, o PROSAD contemplar a saúde do adolescente como um todo, na prática, suas ações têm se concentrado na saúde sexual e reprodutiva dos adolescentes. Outros importantes documentos relacionados à saúde dos adolescentes e jovens são: (a) Diretrizes Nacionais para a Atenção Integral à Saúde dos Adolescentes e Jovens na promoção, proteção e recuperação da saúde (Ministério da Saúde, 2010b), baseadas na Política Nacional de Atenção Integral à saúde de Adolescentes e Jovens (PNAISAJ); e (b) A Linha de cuidado e atenção integral à saúde de crianças, adolescentes e suas famílias em situação de violência (Ministério da Saúde, 2010b). Ambos os documentos reconhecem e orientam profissionais na forma de agir perante os adolescentes e jovens e suas famílias, indicando a necessidade de prevenção em relação à saúde como um todo bem como as situações de violência. $\mathrm{O}$ primeiro documento privilegia a saúde sexual e reprodutiva, o desenvolvimento físico e motor, a prevenção contra as drogas lícitas e ilícitas, embora reconheça a necessidade de prevenção na violência autoinfligida (tentativas de suicídio, por exemplo). Já o segundo documento, atenta para as consequências das formas de violência, reconhecendo a importância de prevenção e intervenção na saúde mental, embora em segundo plano. Dessa forma, a saúde mental parece não ser o foco de programas direcionados a adolescentes, embora essa seja uma necessidade evidenciada através dos estudos revisados.

Nesta revisão teórica, os artigos revelaram as relações entre violência e saúde mental, principalmente, considerando aspectos relativos à doença mental - transtornos internalizantes e externalizantes, TEPT, entre outros. Esses resultados, no entanto, devem ser relativizados, uma vez que podem ser entendidos como reflexos das escolhas metodológicas dos estudos, já que na maioria deles os instrumentos utilizados para medir a saúde mental foram instrumentos que avaliam sintomas de doença mental, embora os estudos tratem de aspectos relativos à saúde mental.

De fato, saúde e doença não são conceitos que possuem um consenso na literatura nacional e internacional, embora haja um esforço na construção de um conceito único e universal de saúde (Boruchovitch \& Mednick, 2002). Através da análise dos estudos e dos instrumentos utilizados, pode-se ressaltar que os artigos apresentam, no geral, uma concepção tradicional de saúde, definindo-a através da ausência de doença, sintomas, sinais ou problemas. Outro conceito também verificado nos estudos foi a definição de saúde pela WHO (2012) que, além de considera-la como a ausência de sintomas - concepção tradicional, acrescenta o estado de bem-estar do sujeito. Esta definição, de certa forma, amplia o conceito, mas não dá conta da complexidade do fenômeno (Boruchovitch \& Mednick, 2002). A definição ecológica de saúde surgiu em meados da década de 60 e 70 com o intuito de dar ênfase às interrelações entre o meio ambiente e a qualidade de vida do sujeito. Nesta concepção, as avaliações relativas à saúde são baseadas nos julgamentos do próprio sujeito sobre a sua saúde - já que essa percepção possui influência sobre suas atitudes e comportamentos - em sua 
capacidade de adaptação perante as situações de doença, sintomas ou eventos estressores. Além do mais, na concepção ecológica, o ambiente social e cultural no qual o indivíduo está inserido também é considerado, já que na definição de normalidade ou saúde são conceitos social e culturalmente construídos (Boruchovitch \& Mednick, 2002).

Ao contrário da maioria dos artigos, quatro estudos (Eggerman \& Panter-Brick, 2010; Epstein-Ngo et al., 2013; Nguyen et al., 2010; Wigderson \& Lynch, 2013) investigados associaram a violência à saúde mental, considerada em uma perspectiva ampla, para além da ausência de doenças. Como contribuições, essas pesquisas indicam que a saúde mental pode ser considerada a partir de uma abordagem de maior alcance, definindo-a não apenas como ausência de doença - como nos estudos que abordam apenas sintomas de doença mental - mas também valorizando aspectos positivos do desenvolvimento dos adolescentes e que podem servir como mediadores das situações de violência, mitigando seus efeitos na saúde mental. De certa forma, estes estudos possuem maior afinidade com uma concepção ecológica de saúde, embora não tenham explicitado suas concepções de saúde e doença (Boruchovitch \& Mednick, 2002).

Alguns estudos investigaram os moderadores das relações entre violência e saúde mental, identificando que alguns fatores podem atenuar ou aumentar os efeitos da exposição à violência. Estratégias de coping, esperança, resiliência, autoestima preservada e bem-estar foram aspectos protetivos ressaltados nos estudos que, quando estão presentes, podem exercer um papel de mediadores, atenuando os efeitos da exposição à violência (Eggerman \& Panter-Brick, 2010; Epstein-Ngo et al., 2013; Nguyen et al., 2010; Wigderson \& Lynch, 2013).

Dessa forma, a maior parte dos artigos possui uma visão tradicional de saúde sem contemplar aspectos da definição da WHO (2012), que considera saúde mental como não meramente a ausência de doenças, mas também um estado de bem-estar. Além disso, poucos ampliam a visão de saúde para uma abordagem ecológica (Bo- ruchovitch \& Mednick, 2002). Ou seja, poucos estudos destacaram aspectos saudáveis do desenvolvimento dos adolescentes ou fatores de proteção que podem moderar os efeitos negativos da exposição à violência. Ainda, as pesquisas não têm destacado os papéis mais amplos, tanto ambientais como sociais e culturais relativos às concepções de saúde. Este achado é importante, sugerindo a relevância de que mais estudos em saúde mental possam considerar aspectos como o bem-estar, por exemplo, ampliando o foco da definição de saúde.

Cabe ainda ressaltar que correlações não indicam causalidade. Portanto, é importante salientar que há uma multiplicidade de fatores envolvidos tanto na exposição à violência quanto relativos aos resultados desenvolvimentais. Deve-se considerar, por exemplo, a forma de exposição à violência (direta ou indireta), o tempo de exposição, bem como o tipo de violência (física, psicológica, sexual ou negligência). Além disso, fatores de proteção podem estar presentes, moderando os efeitos da violência, entre os quais estão rede de apoio, estratégias de coping, alta autoeficácia, boa autoestima, entre outros. Aspectos sociais e culturais, além de econômicos, também são fatores relevantes a serem considerados (Avanci et al., 2007; Koller \& De Antoni, 2004; Mrug \& Windle, 2010). Ainda, no que diz respeito à saúde mental, fatores genéticos também apresentam influência sobre a saúde mental (Avanci et al., 2007; Bahls \& Bahls, 2002; Ministério da Saúde, 1996).

\section{Considerações Finais}

Esta revisão teórica demonstrou como a exposição à violência na adolescência, em vários contextos, está associada a aspectos negativos do desenvolvimento, trazendo à tona a necessidade de maior investimento e sensibilização para essa questão. Sugere, ainda, a necessidade de maior atenção, na adolescência, para a saúde mental, tanto na prevenção de doenças, como na promoção da saúde. No entanto, pode-se observar que a questão é complexa, e que os diversos autores destacam variáveis que exercem influência no impacto da violência aos adolescentes, tais como 
a forma de exposição, questões associadas ao impacto negativo e aspectos relacionados a um enfrentamento positivo (bem-estar, esperança e estratégias de coping). Dessa forma, a problemática da violência e suas repercussões devem ser consideradas como um processo multifacetado.

Dentre as limitações metodológicas citadas nos estudos revisados pode-se citar que alguns dos instrumentos utilizados não são específicos para adolescentes (como, por exemplo, o Conflict Tatics Scales (Haber \& Toro, 2009; Jenkins et al., 2009), Children's Report of Exposure to Violence (Copeland-Linder et al., 2010; Lambert et al., 2012), e que em alguns casos apenas um instrumento foi utilizado para avaliar presença de transtorno mental, como a depressão (Children's Depression Inventory; Bach \& Louw, 2010; Epstein-Ngo et al., 2013; Wigderson \& Lynch, 2013). Além disso, do ponto de vista metodológico, os estudos apresentam delineamento transversal e utilizam questionários com perguntas fechadas, aplicados em um único momento no tempo, o que capta apenas parte da complexidade dos fenômenos tanto da violência como da saúde mental. No caso da violência deveriam ser consideradas suas características, tais como a forma, grau, duração, proximidade com o agressor, além dos fatores pessoais da vítima. No que se refere à saúde mental, características como a ausência de psicopatologias, as quais possuem inúmeros critérios para avaliação, diferentes formas de enfrentamento da violência e aspectos relacionados ao bem-estar deveriam ser considerados, numa abordagem ecológica que permitisse maior amplitude na compreensão do fenômeno.

Além disso, embora não fosse um dos focos dessa revisão teórica, poucos foram os estudos que propuseram estratégias de prevenção ou de intervenção nos casos relativos à violência e saúde mental na adolescência. Identificou-se, após a seleção de artigos, apenas um estudo sobre intervenção em relação à saúde mental (Hasanović et al., 2009). Este aspecto indica um descompasso entre teoria (desenvolvimento científico) e a prática, sugerindo a necessidade de novas propostas de intervenção nos casos de violência e de mais profissionais na área da saúde e humanas, atuando nos diversos contextos de desenvolvimento. As estratégias podem basear-se na identificação dos fatores de risco e de proteção a fim de minimizar a exposição à violência e a doença mental em adolescentes e fortalecer aspectos individuais e contextuais mitigando possíveis riscos. Além disso, é importante que os conhecimentos sobre os direitos e deveres possam chegar até os adolescentes, de forma a empoderá-los na luta contra a exposição às situações de violência e doença mental. Projetos de extensão nas universidades podem dar conta destes aspectos, levando conhecimento até os adolescentes, por exemplo, através da escola, que pode ser um importante ambiente de prevenção e proteção.

A respeito das limitações deste estudo destacam-se questões referentes ao método de revisão sistemática, como a estratégia de busca e critérios de exclusão e inclusão, que podem ter limitado os estudos encontrados, principalmente brasileiros. Poderiam ter sido utilizadas outras palavras-chave relacionadas à violência, tais como abuso físico, psicológico, sexual, entre outras, ampliando o alcance da busca. Além disso, poderiam ser incluídas outras bases de dados, como por exemplo, LILACS, Web of Science e SCOPUS para a ampliação da busca de estudos relativos à temática. Os aspectos ressaltados nessa revisão sugerem a necessidade de trabalhos preventivos e de promoção de saúde acerca da exposição à violência e também em relação à saúde mental dos adolescentes.

\section{Referências}

Al-Krenawi, A., Graham, J. R., \& Kanat-Maymon, Y. (2009). Analysis of trauma exposure, symptomatology and functioning in Jewish Israeli and Palestinian adolescents. British Journal of Psychiatry, 195(5), 427-432. doi:10.1192/bjp. bp.108.050393

Avanci, J. Q., Assis, S. G., Oliveira, R. V. C., Ferreira, R. M., \& Pesce, R. P. (2007). Fatores associados aos problemas de saúde mental em adolescentes. Psicologia: Teoria e Pesquisa, 23(3), 287-294. doi:10.1590/S0102-37722007000300007 
Bach, J. M., \& Louw, D. (2010). Depression and exposure to violence among Venda and Northern Sotho adolescents in South Africa. African Journal of Psychiatry, 13(1), 25-35.

Bahls, S., \& Bahls, F. R. C. (2002). Depressão na adolescência: Características clínicas. Interação em Psicologia, 6(1), 49-57.

Barbosa, P. V., \& Wagner, A. (2013). A autonomia na adolescência: Revisando conceitos, modelos e variáveis. Estudos de Psicologia (Natal), 18(4), 649-658. doi:10.1590/S1413$-294 X 2013000400013$

Benetti, S. P. C., Gama, C., Vitolo, M., Silva, M. B., D’Ávila, A., \& Zavaschi, M. L. (2006). Violência comunitária, exposição às drogas ilícitas e envolvimento com a lei na adolescência. Psico, 37(3), 279-286.

Benetti, S. P. C., Pizetta, A., Schwartz, C. B., Hass, R. A., \& Melo, V. L. (2010). Problemas de saúde mental na adolescência: Características familiares, eventos traumáticos e violência. Psico-USF, 15(3), 321-332.

Betancourt, T. S., Agnew-Blais, J., Gilman, S. E., Williams, D. R., \& Ellis, B. H. (2010). Past horrors, present struggles: The role of stigma in the association between war experiences and psychosocial adjustment among former child soldiers in Sierra Leone. Social Science \& Medicin, 70(1), 17-26. doi: 10.1016 /j.socscimed.2009.09.038

Betancourt, T. S., Brennan, R. T., Rubin-Smith, J., Fitzmaurice, G. M., \& Gilman, S. E. (2010). Sierra Leone's former child soldiers: A longitudinal study of risk, protective factors and mental health. Journal of the American Academy of Child and Adolescent Psychiatry, 49(6), 606615. doi:10.1016/j.jaac.2010.03.008

Boruchovitch, E., \& Mednick, B. R. (2002). The meaning of health and illness: Some considerations for health psychology. Psico-USF, 7(2), 175183. doi: $10.1590 / \mathrm{S} 1413-82712002000200006$

Braga, L. L., \& Dell'Aglio, D. D. (2012). Exposição à violência de adolescentes de diferentes contextos: Família e instituições. Estudos de Psicologia (Natal), 17(3), 413-420. doi:10.1590/S1413$-294 X 2012000300009$

Cano, P., Gutiérrez, C., \& Nizama, M. (2009). Tendencia a la violência e ideación suicida em adolescentes escolares em una ciudad de La Amazônia peruana. Revista Peruana de Medicina Experimental y Salud Publica, 26(2), 175-181.
Copeland-Linder, N., Lambert, S. F., \& Ialongo, N. S. (2010). Community violence, protective factors, and adolescent mental health: A profile analysis. Journal of Clinical Child \& Adolescent Psychology, 39(2), 176-86. doi:10.1080/15374410903532601

Eggerman, M., \& Panter-Brick, C. (2010). Suffering, hope, and entrapment: Resilience and cultural values in Afghanistan. Social Science \& Medicine, 71(1), 71-83. doi:10.1016/j.socscimed.2010.03.023

Epstein-Ngo, Q., Maurizi, L. K., Bregman, A., \& Ceballo, R. (2013). In response to community violence: Coping strategies and involuntary stress responses among Latino adolescents. $\mathrm{Cul}$ tural Diversity and Ethnic Minority Psychology, 19(1), 38-49. doi:10.1037/a0029753

Guzmán, M. L. M. (2007). Mirando al futuro: desafíos y oportunidades para el desarrollo de los adolescentes en Chile. Psykhe, 16(1), 3-14. doi:10.4067/S0718-22282007000100001

Haber, M. G., \& Toro, P. A. (2009). Parent-adolescent violence and later behavioral health problems among homeless and housed youth. American Journal of Orthopsychiatry, 79(3), 305-318. doi: $10.1037 / \mathrm{a} 0017212$

Habigzang, L. F., \& Caminha, R. M. (2004). Abuso sexual contra crianças e adolescentes: Conceituação e intervenção clínica. São Paulo, SP: Casa do Psicólogo.

Hasanović, M., Srabović, S., Rasidović, M., Sehović, M., Hasanbasić, E., Husanović, J., \& Hodzić, R. (2009). Psychosocial assistance to students with posttraumatic stress disorder in primary and secondary schools in post-war Bosnia Herzegovina. Psychiatria Danubina, 21(4), 463-473. doi:10.1016/S0924-9338(12)74465-6

Haynie, D., Petts, R. J., Maimon, D., \& Piquero, A. R. (2009). Exposure to violence in adolescence and precocious role exits. Journal Youth Adolescence, 38(3), 269-286. doi:10.1007/s10964-0089343-2

Houck, C. D., Nugent, N. R., Lescano, C. M., Peters, A., \& Brown, L. K. (2010). Sexual abuse and sexual risk behavior: Beyond the impact of psychiatric problems. Journal of Pediatric Psychology, 35(5), 473-483. doi:10.1093/jpepsy/jsp111

Instituto Brasileiro de Geografia e Estatística. (2009). Pesquisa Nacional de Saúde do Escolar-PeN$S E$. Rio de Janeiro, RJ: Autor. 
Jenkins, E. J., Turner, L., \& Wang, E. (2009). Traumatic events involving friends and family members in a sample of African early adolescents. American Journal of Orthopsychiatry, 79(3), 398-406. doi:10.1037/a0016659

Koller, S. H., \& De Antoni, C. (2004). Violência Intrafamiliar: Uma visão ecológica. In S. Koller (Ed.), Ecologia do desenvolvimento humano: Pesquisa e Intervenção no Brasil (pp. 293-310). São Paulo, SP: Casa do Psicólogo.

Lambert, S. F., Cammack, N. L., Boyd, R. C., \& Ialongo, N. S. (2012). Relationship proximity to victims of witnessed community violence: Associations with adolescent internalizing and externalizing behaviors. American Journal of Orthopsychiatry, 82(1), 1-9. doi:10.1111/j.19390025.2011.01135.x

Lopes, A. L. M., \& Fracolli, L. A. (2008). Revisão sistemática de literatura e metassíntese qualitativa: Considerações sobre sua aplicação na pesquisa em enfermagem. Texto \& Contexto Enfermagem, 17(4), 771-778. doi:10.1590/S010407072008000400020

Malta, D. C., Souza, E. R., Silva, M. M. A., Silva, C. S., Andreazzi, M. A. R., Crespo, C., \& Penna, G. O. (2010). Vivência de violência entre escolares brasileiros: Resultados da Pesquisa Nacional de Saúde do Escolar (PeNSE). Ciência \& Saúde Coletiva, 15(2), 3053-3063. doi:10.1590/S141381232010000800010

Ministério da Saúde. (1996). Programa Saúde do Adolescente. Bases Programáticas (2. ed.). Brasília, DF: Autor.

Ministério da Saúde. (2010a). Linha de cuidado para a atenção integral à saúde de crianças, adolescentes e suas famílias em situação de violência. Brasília, DF: Autor.

Ministério da Saúde. (2010b). Diretrizes nacionais para a atenção integral à saúde de adolescentes e jovens na promoção, proteção e recuperação da saúde. Brasília, DF: Autor.

Ministério da Saúde. (2011). Viva: Instrutivo de notificação de violência doméstica, sexual e outras violências. Brasília, DF: Autor.

Mrug, S., \& Windle, M. (2010). Prospective effects of violence exposure across multiple contexts on early adolescents' internalizing and externalizing problems. Journal of Child Psychology and Psychiatry, 51(8), 953-961. doi:10.1111/j.14697610.2010.02222.x
Nguyen, H. T., Dunne, M. P., \& Vu Le, A. (2010). Multiple types of child maltreatment and adolescent mental health in Viet Nam. Bulletin World Health Organization, 88(1), 22-30. doi:10.2471/ BLT.08.060061

Rheingold, A. A., Zinzow, H., Hawkins, A., Saunders, B. E., \& Kilpatrick, D. G. (2012). Prevalence and mental health outcomes of homicide survivors in a representative US sample of adolescents: Data from the 2005 National Survey of Adolescents. Journal Child Psychology Psychiatry, 53(6), 687-694. doi:10.1111/j.14697610.2011.02491.x

Rocha, T. B., Zeni, C. P., Caetano, S. C., \& Kieling, C. (2013). Mood disorders in childhood and adolescence. Revista Brasileira de Psiquiatria, 35(1), 22-31. doi:10.1590/1516-4446-2013-S106

Rutter, M. (1999). Resilience concepts and findings: Implications for family therapy. Journal of $\mathrm{Fa}$ mily Therapy, 21, 119-144.

Sampaio, R. F., \& Mancini, M. C. (2007). Estudos de revisão sistemática: Um guia para síntese criteriosa da evidência científica. Brazilian Journal of Physical Therapy, 11(1), 83-89.

Steinberg, L. (2000). The family at adolescence: Transition and transformation. Journal of Adolescent Health, 27, 170-178.

Steinberg, L. (2005). Adolescence ( $7^{\text {th }}$ ed.). Boston, MA: McGraw-Hill.

Steinberg, L., \& Lerner, R. M. (2004). The scientific study of adolescence: A brief history. The Journal of Early Adolescence, 24(1), 45-54.

United Nation Educational, Scientific and Cultural Organization. (2011). O direito de ser adolescente: Oportunidade para reduzir vulnerabilidades e superar desigualdades. Brasília, DF.

Voisin, D. R., \& Neilands, T. B. (2010). Community violence and health risk factors among adolescents on Chicago's outside: Does gender matter? Journal Adolescent Health, 46(6), 600-602. doi:10.1016/j.jadohealth.2009.11.213

Waiselfisz, J. J. (2012). Mapa da violência 2012: Crianças e adolescentes do Brasil. Rio de Janeiro, RJ: Cebela.

Walsh, S. D., Molcho, M., Craig, W., Harel-Fisch, Y., Huynh, Q., Kukaswadia, A., \& Pickett, W. (2013). Physical and emotional health problems experienced by youth engaged in physical fighting and weapon carrying. PLoS One, 8(2), 1-8. doi:10.1371/journal.pone.0056403 
Wigderson, S., \& Lynch, M. (2013). Cyber and traditional peer victimization: Unique relationships with adolescent well-being. Psychology of Violence, 3(4), 297-309. doi:10.1037/a0033657

World Health Organization. (2002). Version of the Introduction to the World Report on Violence and Health. Geneva, Switzerland: Author. Retrieved from http://www.who.int/violence injury_prevention/violence/world_report/en/introduction.pdf

World Health Organization. (2010). Mental health strengthening our response. Fact sheet 220. Retrieved from http://www.who.int/mediacentre/ factsheets/fs220/en/

World Health Organization. (2012). Adolescent mental health: Mapping actions of nongovernmental organizations and other international development organizations. Geneva, Switzerland: Author. Retrieved from http://www.who.int/ mental_health/publications/adolescent_mental_ health/en/index.html

World Health Organization. (2014). Health for the world's adolescents: A second chance in the second decade. Retrieved from http://apps.who. int/adolescent/second-decade

Yunes, M. A. M. (2003). Positive psychology and resilience: Focus on the individual and families. Psicologia em Estudo, 8, 75-84. doi:10.1590/S1413-73722003000300010

Zappe, J. G., \& Dias, A. C. G. (2010). Delinquência juvenil na produção científica nacional: Distância entre achados científicos e intervenções concretas. Barbarói, (33), 82-103.
Zappe, J. G., \& Dias, A. C. G. (2012). Violência e fragilidades nas relações familiares: Refletindo sobre a situação de adolescentes em conflito com a lei. Estudos de Psicologia (Natal), 17(3), 389395. doi:10.1590/S1413-294X2012000300006

Zavaschi, M. L., Benetti, S., Polanczyk, G. V., Soles, N., \& Sanchotene, M. L. (2002). Adolescents exposed to physical violence in the community: A survey in Brazilian public schools. Revista Panamericana de Salud Publica, 12(5), 327332.

Zinzow, H. M., Ruggiero, K. J., Resnick, H., Hanson, R., Smith, D., Saunders, B., \& Kilpatrick, D. (2009). Prevalence and mental health correlates of witnessed parental and community violence in a national sample of adolescents. Journal of Child Psychology and Psychiatry, 50(4), 441450. doi:10.1111/j.1469-7610.2008.02004.x

Zoltowski, A. P. C., Costa, A. B., Teixeira, M. A. P., \& Koller, S. H. (2014). Qualidade metodológica das revisões sistemáticas em periódicos de psicologia brasileiros. Psicologia: Teoria e Pesquisa, 30(1), 97-104. doi:10.1590/S010237722014000100012
Recebido: 21/10/2014

$1^{a}$ revisão: 19/01/2015

Aceite final: 10/02/2015 